\title{
Extended Capabilities of the 3-D Smith Chart With Group Delay and Resonator Quality Factor
}

\author{
Andrei A. Muller, Member, IEEE, Esther Sanabria-Codesal, Alin Moldoveanu, \\ Victor Asavei, and Stepan Lucyszyn, Fellow, IEEE
}

\begin{abstract}
This paper extends the capabilities of the 3-D Smith chart for representing positive and negative differential-phase group delay and the associated loaded resonator quality factor, displayed simultaneously with scattering (S)-parameters. Here, mathematical concepts, inspired from elementary differential geometry and topology, are used to implement 3-D projections. It is shown that a condition for a circuit to exploit negative differential-phase group delay is that its $S$-parameter winding number should be $\geq 0$ (relative to its origin). Finally, exemplar network responses that exhibit both positive and negative differential-phase group delay and loaded resonator quality factor are shown with the 3-D Smith chart. The convenience of being able to simultaneously display a wider range of parameters on one visualization platform, with the 3-D Smith chart, may help to speed-up the design and analysis of microwave circuits by the user.
\end{abstract}

Index Terms-Computer-aided design (CAD), differentialphase group delay, negative group delay (NGD), non-Foster, quality factor, Smith chart.

\section{INTRODUCTION}

G ROUP delay represents the time taken for the amplitude envelope of a carrier signal (or information) to be transmitted between two points. Ideally, group delay is constant with frequency, across a band-limited channel, such that the Fourier components of the information at the transmit point experience the same delay and combine to recreate the original information at the receive point. In practice, however, the end-to-end group delay of a system is seldom constant with frequency across the band-limited channel.

As a result, to mitigate against signal distortion, group delay equalization (across the bandwidth of a channel) is often

Manuscript received April 13, 2016; revised August 15, 2016 and September 19, 2016; accepted September 23, 2016. The work of A. A. Muller was supported under SIWTUNE Marie Curie Integration Grant 322162. The work of E. Sanabria-Codesal was supported in part under DGCYT Grant MTM2015-64013-P.

A. A. Muller is with the Microwave Application's Group-i-Team, Universidad Polithenica de Valencia, 46022 Valencia, Spain (e-mail: andrei.stefan1@gmail.com).

E. Sanabria-Codesal is with the Applied Mathematics Department, Universidad Polithenica de Valencia, 46022 Valencia, Spain (e-mail: esanabri@mat.upv.es)

A. Moldoveanu and V. Asavei are with the Faculty of Automatic Control and Computers, University Politehnica Bucharest, 060042 Bucharest, Romania (e-mail: alin.moldoveanu@cs.pub.ro; victor.asavei@cs.pub.ro).

S. Lucyszyn is with the Department of Electrical and Electronic Engineering, Imperial College London, London SW7 2AZ, U.K. (e-mail: s.lucyszyn@imperial.ac.uk).

Color versions of one or more of the figures in this paper are available online at http://ieeexplore.iee.org.

Digital Object Identifier 10.1109/TMTT.2016.2614931

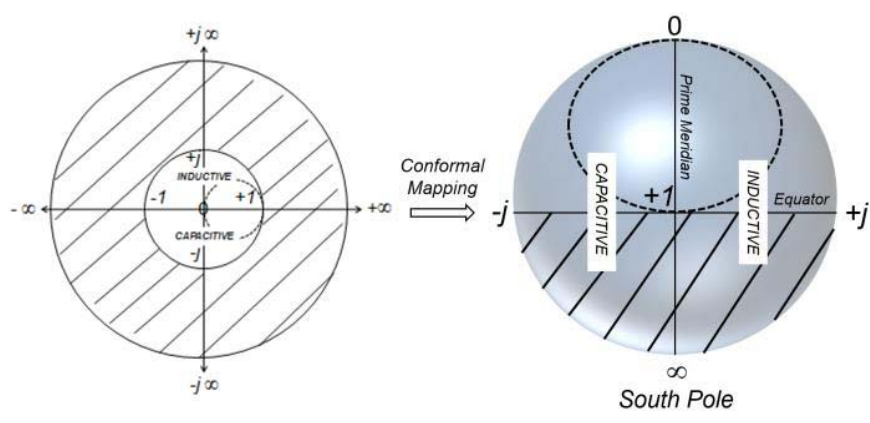

Fig. 1. Conformal mapping from 2-D to 3-D Smith charts (impedance version)

employed, especially close to the cutoff frequencies of the channel filter, where there may be significant peaks in group delay. Tunable differential-phase negative group delay (NGD) circuits operating at microwave frequencies were first introduced by Lucyszyn et al. [1], [2] over two decades ago. Also known as NGD networks, in recent years they have attracted interest from the microwave community [3]-[8].

The 3-D Smith chart (impedance version) was recently introduced by Muller et al. [9]-[13] in 2011. This visualization platform represents the response of passive components/networks, active devices/ circuits, and mixed topology subsystems, by exploiting the unity radius Riemann sphere, using the conformal mapping illustrated in Fig. 1.

In terms of voltage-wave reflection and transmissions coefficients, the Northern hemisphere represents a modulus less than unity, while the Southern hemisphere represents a modulus greater than unity-the equator represents the pure reactance unity radius circle of the 2-D Smith chart. The Eastern hemisphere represents inductance and West capacitance-separated by the prime meridian that represents pure resistance (positive and negative in the Northern and Southern hemispheres, respectively). Therefore, the origin of the 2-D Smith chart is mapped to the North Pole of the 3-D Smith chart, while infinite is just as conveniently mapped to the South Pole [9], [10].

Until very recently, the main applications for the 3-D Smith chart included complex port matching, representing amplifier stability circles (originally at a single frequency, it will be shown in the Appendix that this can also be extended to cover a wide frequency range) and oscillator design, by projecting onto the surface only of the sphere [9]-[11], with computer-aided design (CAD) software now commercially available [12]. The basic geometrical framework employs a 
stereographical projection to map the generalized Smith chart onto the Riemann sphere.

In 2014, Muller et al. [13] proposed the use of the third dimension of the Euclidean plane to plot the unilateral transducer constant power gain circles outside the 3-D chart. Here, a simple projection (with a constant scaling factor) from the center of the sphere (i.e., the origin of the 3-D Smith chart) maps the unilateral transducer power gain circles in 3-D space.

The capability of the 3-D Smith chart to provide intuitive insights into phenomena that would be otherwise difficult to visualize on a 2-D Smith chart [14] will be further exploited here. Mathematical concepts (inspired from elementary differential geometry and topology) will bring to light simple and straightforward models for visualizing not only the scattering (S)-parameters but also the associated differential-phase group delay and loaded resonator quality (Q)-factor. For example, a one-to-one mapping between these parameters allows the viewer to visualize the forward voltagewave transmission coefficient $S_{21}$ on the 3-D Smith chart, the associated differential-phase group delay in 3-D space, and the corresponding $\mathrm{Q}$-factor for an equivalent series $R L C$ resonator along the normal plane of the 3-D differential-phase group delay space curve.

This paper first introduces basic concepts from differential geometry [15] (the branch of mathematics that studies the geometry of curves and surfaces in 3-D space) to define the space curve that can represent both transmission and reflection response group delays and associated loaded resonator Q-factor (thickened curve). Then a condition is presented, based on the notion of a winding number that a circuit has to obey in order to exhibit negative differential-phase group delay within the displayed frequency range. Finally, several network exemplars are considered, demonstrating the potential for the visualization of multiple parameters in 3-D.

In Section II, given $S_{21}(\omega)$, where $\omega$ represents angular frequency, a new function $\tau_{3 D}(\omega)$ is defined in the form of a space curve having its modulus being proportional to the differential-phase group delay and the phase of $S_{21}(\omega)$. For the first time, the exterior of the 3-D Smith chart (outer space) will be used to display positive values of differential-phase group delay, while its interior (inner space) displays negative values; this is not possible on a conventional 2-D Smith chart. The theory is developed for the concept of transmission response group delay, but similar implementation and results are also found with reflection response group delay (e.g., as exploited by termination impedances in reflection-type topologies [1], [2]). Since the differential-phase group delay curve is no longer a plane curve, basic elements of differential geometry and 3-D projections can be exploited [16]. Here, the loaded resonator Q-factor associated with differential-phase group delay is plotted as a generalized cylinder [17], [18] of variable radius in the normal plane of the differential-phase group delay curve, tracking its motion (as frequency changes). The value of loaded resonator Q-factor at each frequency point will thus be given by the radius of the generalized cylinder (obtained by extruding a circle of nonconstant radius along the path of the differential phase group delay curve).

\section{Differential Geometry Formulation}

\section{A. Differential-Phase Group Delay Based on Homothety}

Transmission parameter $S_{21}(\omega)$ can be defined using either the Cartesian (1) or exponential (2) forms, where $a(\omega)$ and $b(\omega)$ represent its real and imaginary parts, respectively,

$$
\begin{aligned}
S_{21}(\omega) & =a(\omega)+j b(\omega) \\
S_{21}(\omega) & =\left|S_{21}(\omega)\right| e^{j \angle S_{21}(\omega)} \\
\left|S_{21}(\omega)\right| & =\sqrt{a(\omega)^{2}+b(\omega)^{2}} \\
\angle S_{21}(\omega) & =\tan ^{-1}\left\{\frac{b(\omega)}{a(\omega)}\right\}
\end{aligned}
$$

where $j=\sqrt{-1}$ is the complex operator. The corresponding differential-phase group delay $\tau(\omega)$ of a network can be defined as the negative derivative of insertion phase $\angle S_{21}(\omega)$ with respect to angular frequency [1], [2]

$$
\tau(\omega)=-\frac{\partial \angle S_{21}(\omega)}{\partial \omega} .
$$

Clearly, differential-phase group delay will be negative when the phase-frequency gradient is positive (without breaking the law of causality), which is often found in lossy scenarios.

In principle, the 2-D differential-phase group delay function $\tau_{2 D}(\omega)$ can be defined as having its amplitude and phasor given by (3) and in (2), respectively,

$$
\tau_{2 D}(\omega)=\tau(\omega) e^{j \angle S_{21}(\omega)} .
$$

However, in practice, it will be observed that the $\tau_{2 D}(\omega)$ curve cannot be defined in exponential form, since (3) is not always positive. Fortunately, the 3-D Smith chart can be exploited using the following steps. The differential-phase group delay is first normalized to its maximum absolute peak value (to achieve a normalized value within the interval $[-1,1])$

$$
\tau_{N}(\omega)=\frac{\tau(\omega)}{|\tau(\omega)| \operatorname{MAX}} .
$$

The transmission parameter is plotted onto the 3-D Smith chart, to give a curve of $S_{\mathbf{2 1 3}}(\boldsymbol{\omega})$ as frequency changes on the surface of the Riemann sphere [9], [10] using the following mapping function [9, eq.(3)], written here in Cartesian vector form:

$$
\begin{aligned}
\boldsymbol{S}_{\mathbf{2 1 3 D}}(\omega)= & \frac{2 a(\omega)}{1+\left|S_{21}(\omega)\right|^{2}} i+\frac{2 b(\omega)}{1+\left|S_{21}(\omega)\right|^{2}} j \\
& \left.+\frac{1-\left|S_{21}(\omega)\right|^{2}}{1+\left|S_{21}(\omega)\right|^{2}} k\right)
\end{aligned}
$$

where $\boldsymbol{i}, \boldsymbol{j}$, and $\boldsymbol{k}$ represent the orthogonal versors with respect to the center of the Riemann sphere. The $S_{\mathbf{2 1 3}}(\boldsymbol{\omega})$ curve is traced by the position vectors, at each frequency point, relative to the sphere's origin.

Now, a variable homothety (a bijection transformation obtained by pushing or pulling a curve along a line segment formed by the points of the curve and a fixed point-in our case the center of the 3-D Smith chart) [18] is considered alongside the associated scattering (S)-parameter $S_{\mathbf{2 1 3}}(\boldsymbol{\omega})$, with factor $\left(1+\tau_{N}(\omega)\right)$. This factor represents a dilation of the $S_{\mathbf{2 1 3 D}}(\omega)$ curve, relative to the surface of the sphere. This 


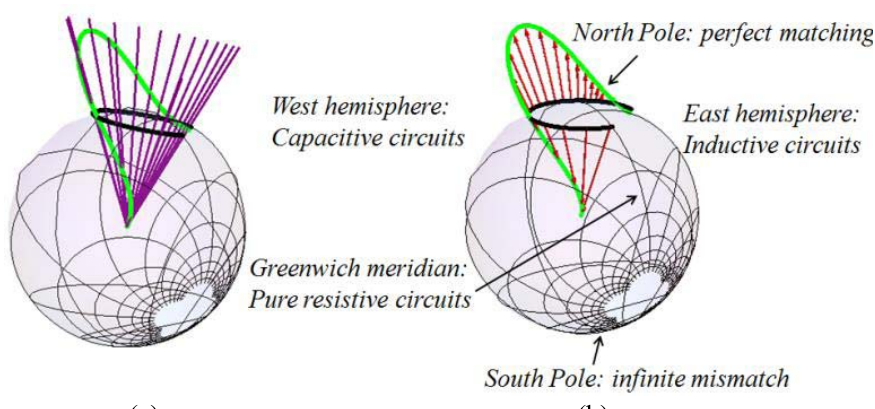

(a)

(b)

Fig. 2. Arbitrary example. (a) Radial lines (each representing a discrete frequency) emanating from the sphere's origin through the surface curve of $S_{213 D}(\omega)$ and 3-D space curve of $\tau_{3 D}(\omega)$. (b) Vector relationship between $S_{213 D}(\omega)$ and $\tau_{3 D}(\omega)$, when the latter becomes negative an observer on the $S_{213 D}(\omega)$ curve looks toward the interior of the 3-D Smith chart to follow its path.

defines a space curve $\tau_{3 D}(\omega)$ that can be exterior or interior to the unity radius $3-\mathrm{D}$ chart, which is a bijective function to $S_{213 D}(\omega)$

$$
\boldsymbol{\tau}_{\mathbf{3 D}}(\boldsymbol{\omega})=\left(1+\tau_{N}(\omega)\right) \boldsymbol{S}_{\mathbf{2 1 3} D}(\boldsymbol{\omega}) .
$$

In this way, (7) keeps the phase of $\tau_{2 D}(\omega)$ and $S_{21}(\omega)$ with the 3-D Smith chart and makes it possible to visualize the corresponding normalized value $\tau_{N}(\omega)$ using the exterior and interior of the 3-D Smith chart for positive and negative values, respectively. At each frequency point, a radial line emanates from the sphere's origin toward the surface projection of $S_{\mathbf{2 1 3}}(\omega)$. Moving along the same radial line, an observer at the sphere's origin can also visualize $\tau_{3 D}(\omega)$, as illustrated in Fig. 2(a). For the observer, there is no difference between (6) and (7), as the radial line emanating from the observer touches both curves.

The basic mathematical tool used in (7) is based on homothety. The curves from (6) and (7) are topologically identical. One can think of the curve from (7) as being pulled-out if the differential-phase group delay is positive or pushed-in if negative, along the transmission parameter curve; with a variable weighting $\left(1+\tau_{N}(\omega)\right)$ directed either away from or toward the sphere's origin, respectively.

With $S_{213 D}(\omega)$, the observer can directly read off $S_{21}(\omega)$ from the surface of the sphere. However, this is not the case with $\tau(\omega)$, as $|\tau(\omega)| \operatorname{MAX}$ is not indicated. The distance $d$ from $S_{213 D}(\omega)$ to $\tau_{3 D}(\omega)$, along a radial line emanating from the sphere's origin, is given by

$$
d\left(\boldsymbol{S}_{\mathbf{2 1 3} D}(\boldsymbol{\omega}), \boldsymbol{\tau}_{3 D}(\boldsymbol{\omega})\right)=\left|\tau_{N}(\omega)\right|
$$

It can be seen that if $\tau_{N}(\omega)>0$ there will be an exterior mapping, while $\tau_{N}(\omega)<0$ will have interior mapping. The second factor in (7) has values in the interval [0,2]. Therefore, this factor will be zero when $\tau_{3 D}(\omega)$ reaches the minimum negative value (i.e., at the sphere's origin), unity at the points where it vanishes (i.e., on the surface of the sphere) and 2 when it reaches the maximum positive values (i.e., exterior with unity distance from the surface of the sphere).

\section{B. Frenet Coordinate System}

When working with curves in 3-D space, instead of using the Cartesian form, a coordinate system can be adapted to

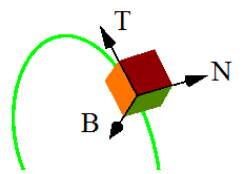

Fig. 3. Frenet frame showing the osculating, normal, and rectifying planes for the $\tau_{3 D}(\omega)$ curve. $T(\omega)$ points in the direction of increasing frequency.

the local structure, which moves with an observer along its trajectory, at each point along the curve. The Frenet moving frame is a more meaningful vector system that provides a coordinate system at each frequency point [19], [20], tracking the motion of $\tau_{3 D}(\omega)$ as frequency changes. For this, $\tau_{3 D}(\omega)$ is first rewritten, by putting (6) into (7), as

$$
\begin{aligned}
& \boldsymbol{\tau}_{3 D}(\omega) \\
& =\left(1+\tau_{N}(\omega)\right) \\
& \quad \times\left(\frac{2 a(\omega)}{1+\left|S_{21}(\omega)\right|^{2}} i+\frac{2 b(\omega)}{1+\left|S_{21}(\omega)\right|^{2}} j+\frac{1-\left|S_{21}(\omega)\right|^{2}}{1+\left|S_{21}(\omega)\right|^{2}} k\right) .
\end{aligned}
$$

In the Frenet coordinate system, defined by the unit vectors $\{\boldsymbol{T}(\omega), N(\omega), B(\omega)\}$, the tangent unit vector $\boldsymbol{T}(\omega)$ represents the derivative of $\tau_{3 D}(\omega)$ with respect to angular frequency, normalized by its modulus, and is defined as

$$
\boldsymbol{T}(\omega)=\frac{\boldsymbol{\tau}_{3 D}^{\prime}(\omega)}{\left|\boldsymbol{\tau}_{3 D}^{\prime}(\omega)\right|}
$$

where the tick mark' denotes the derivative with respect to $\omega$. The normal unit vector $N(\omega)$ has the corresponding derivative of the tangent unit vector

$$
\boldsymbol{N}(\boldsymbol{\omega})=\frac{\boldsymbol{T}^{\prime}(\omega)}{\left|\boldsymbol{T}^{\prime}(\omega)\right|} .
$$

$\boldsymbol{T}(\boldsymbol{\omega})$ points in the forward direction of travel (as frequency increases), while $N(\omega)$ points in the direction in which $\tau_{3 D}(\omega)$ is turning [20]. The tendency of $\tau_{3 D}(\omega)$ to twist out of the plane created by the tangent and normal unit vectors, in the direction perpendicular to this plane, is reflected by the binormal unit vector $\boldsymbol{B}(\boldsymbol{\omega})$, defined by the vector cross product

$$
B(\omega)=T(\omega) \times N(\omega) .
$$

All three unit vectors are mutually orthogonal and define a coordinate system [15], [16], [20] along $\tau_{3 D}(\omega)$; this can be thought of as a spaceship-based coordinate system. The plane formed by $N(\omega)$ and $T(\omega)$ is called the osculating plane and moves around the $\tau_{3 D}(\omega)$ curve; the plane formed by $\boldsymbol{B}(\omega)$ and $N(\omega)$ is called the normal plane and is perpendicular to $\tau_{3 D}(\omega)$; and the rectifying plane is formed by $\boldsymbol{T}(\omega)$ and $\boldsymbol{B}(\boldsymbol{\omega})$. These planes are illustrated in Fig. 3.

\section{Loaded Resonator Quality Factor}

There is a convenient relationship between the differentialphase group delay and the loaded quality factor of an equivalent series or shunt $R L C$ resonator at its undamped (or driven) angular resonance frequency $\omega_{o}[21]$

$$
Q_{\mathrm{LR}}\left(\omega_{o}\right)=\frac{\omega_{o}}{2} \cdot\left|\tau\left(\omega_{o}\right)\right| .
$$




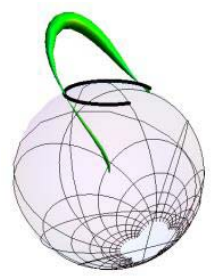

Fig. 4. Qualitative display of loaded resonator Q-factor in the normal plane of the differential-phase group delay curve given in Fig. 2, creating a generalized cylinder of radius proportional to (15).

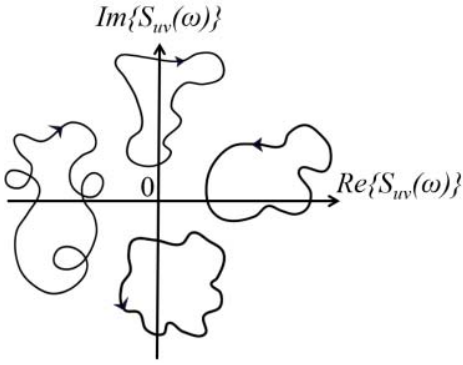

(a)

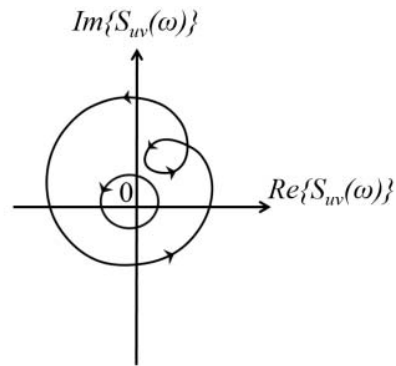

(b)
Fig. 5. Arbitrary loci on the 2-D Smith chart. (a) Four NGD networks exhibiting a zero S-parameter winding number. (b) Two NGD networks exhibiting a unity S-parameter winding number. With the inner loop, the network exhibits negative differential-phase group delay at all the frequencies displayed, while with the outer loop the associated network has a more bandlimited performance.

Note that the network is inherently loaded by the reference impedance of the system and/or any other impedance(s) that terminate its ports. Therefore, the level of port loading will directly affect the differential-phase group delay and associated loaded resonator Q-factor.

Using (13), the resonator's loaded quality factor at resonance $Q_{\mathrm{LR}}\left(\omega_{o}\right)$ can be superimposed onto the normal plane to the $\tau_{3 D}(\omega)$ curve (offering a more aesthetic perspective [16]). Hence, taking advantage of the 3-D representation of the $\tau_{3 D}(\omega)$ curve (7), the normal plane of the curve can be used (as in [16]-[18]) to display this Q-factor as a thickened curve $Q_{3 D}(\omega, \theta)$ at each frequency point. Here, a variable radius cylinder is defined, based on the normalized loaded resonator Q-factor along the curve, by using the following parameterization with $\boldsymbol{\tau}_{\mathbf{3 D}}(\boldsymbol{\omega})$ :

$$
\begin{aligned}
\boldsymbol{Q}_{3 D}(\boldsymbol{\omega}, \boldsymbol{\theta})= & \boldsymbol{\tau}_{3 D}(\boldsymbol{\omega}) \\
& +[\boldsymbol{N}(\boldsymbol{\omega}) \cos \theta+\boldsymbol{B}(\boldsymbol{\omega}) \sin \theta] Q_{N}(\boldsymbol{\omega})
\end{aligned}
$$

where $0 \leq \theta \leq 2 \pi$ is an angle that defines a circle [22] around each frequency point along the $\tau_{3 D}(\omega)$ curve, to create a surface; $Q_{N}(\omega)$ is the normalized loaded resonator Q-factor with an additional arbitrary scaling factor of 5

$$
Q_{N}(\omega)=\frac{Q_{\mathrm{LR}}(\omega)}{\left.5 \cdot Q_{\mathrm{LR}}(\omega)\right|_{\mathrm{MAX}}} .
$$

Using (14) and (15), Fig. 4 qualitatively shows how loaded resonator Q-factor can be displayed with a 3-D Smith chart for the same differential-phase group delay curve given in Fig. 2.

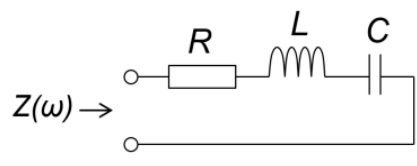

Fig. 6. One-port series $R L C$ network exhibiting negative differential-phase group delay (reflection response).

\section{S-Parameter Winding Numbers}

The winding number of a closed curve $\chi$ in the plane around a given point $p_{0}$ is the integer that represents the total number of times that the curve $\chi$ rotates completely around the point $p_{0}$. The winding number depends on the orientation of the curve and is negative if the curve travels clockwise around the point $p_{0}$. This mathematical concept plays an important role in many disciplines (e.g., algebraic topology, vector calculus, complex analysis, and physics) [23].

For an observer located at the origin of the 2-D Smith chart [i.e., the origin of the $S_{u v}(\omega)$ plane, where $u, v \in$ $[1,2]$ for the one- or two-port network examples used in this paper], the differential-phase group delay is negative as the observer rotates in a counterclockwise direction and positive in a clockwise direction. Therefore, from an observer tracking the movement with frequency of an S-parameter loop, the associated winding number is defined as the number of times $S_{u v}(\omega)$ rotates completely around the origin in a counterclockwise direction. A condition for a circuit to exhibit negative differential-phase group delay is that it has a zero or positive S-parameter winding number.

Fig. 5(a) and (b) shows arbitrary examples of zero and unity S-parameter winding numbers, respectively. In all but one case, the observer has to rotate in both clockwise and counterclockwise directions in order to track the movement of the closed loop, indicating that $\tau(\omega)<0$ over just part of the fully displayed frequency range. Examples of classical NGD networks will be given in Sections III and IV.

\section{ZERo S-PARAMETER WiNDing NUMBER}

The one-port series $R L C$ network shown in Fig. 6 can exhibit negative differential-phase group delay (reflection response) when $R>Z_{o}$ [1]-[3], where $Z_{o}$ is the (real) reference impedance of the system. From Fig. 6, the voltagewave reflection coefficient is given as

$$
S_{11}(\omega)=\frac{Z(\omega)-Z_{0}}{Z(\omega)+Z_{0}}
$$

where $Z(\omega)$ is the driving point impedance of the network, given by

$$
Z(\omega)=R+j\left(\omega L-\frac{1}{\omega C}\right) .
$$

Since $R, L$, and $C$ have positive fixed values, geometrically (17) defines an extended line in the complex impedance plane that passes through $Z=R$ in the right half of the impedance plane. This extended line is mapped by (16) into a circle onto the 2-D Smith chart (because (16) is a Möbius transformation), passing through $S_{11}\left(\omega_{o}\right)=\left(R-Z_{0}\right) /\left(R+Z_{0}\right)$ and $S_{11}(\omega)=1$ at dc and infinite frequency. For example, 


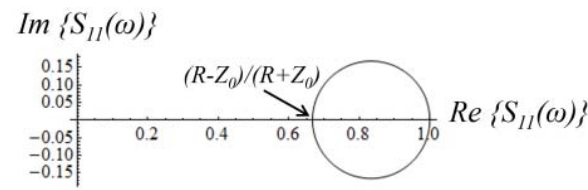

Fig. 7. Voltage-wave reflection coefficient $S_{11}(\omega)$ loop on the 2-D Smith chart for the $R L C$ network given in Fig. 6, having a zero S-parameter winding number when $R>Z_{o}$, exhibiting negative differential-phase group delay.

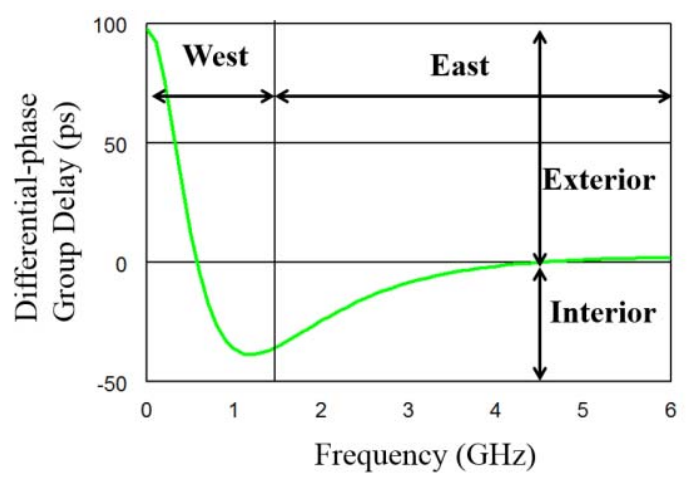

Fig. 8. Calculated differential-phase group delay (reflection response) for the one-port network given in Fig. 6.

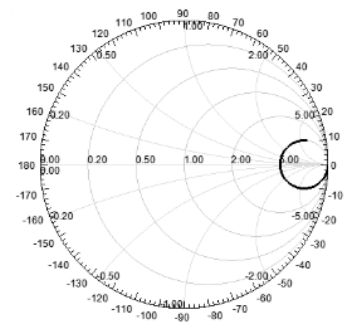

(a)

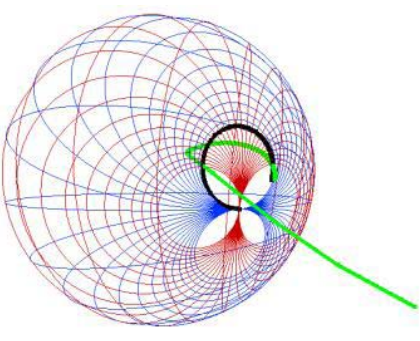

(b)
Fig. 9. Smith chart displays of calculated voltage-wave reflection coefficient (surface curve) for the network in Fig. 6. (a) 2-D chart with $S_{11}(\omega)$ corresponding to Fig. 7. (b) 3-D chart with $S_{113 D}(\omega)$ and reflection response $\tau_{3 D}(\omega)$ curve (3-D space curve).

with arbitrary values of $L=10 \mathrm{nH}, C=1 \mathrm{pF}$, and $R=$ $250 \Omega$, with $Z_{0}=50 \Omega$, the calculated $S_{11}(\omega)$ and associated differential-phase group delay are shown in Figs. 7 and 8 , respectively.

Fig. 9(a) shows a section of the calculated loop of $S_{11}(\omega)$ in the 2-D Smith chart, while Fig. 9(b) shows the corresponding differential-phase group delay with the 3-D Smith chart. As predicted by (7) and seen in Fig. 8, the $\boldsymbol{\tau}_{3 D}(\omega)$ curve is interior from 0.57 to $4.46 \mathrm{GHz}$. Moreover, below, at, and above the undamped resonance frequency of $1.592 \mathrm{GHz}$, the network changes from being capacitive to resistive to inductive, respectively. As a result, the movement with frequency of $\tau_{3 D}(\omega)$ starts above the Western hemisphere and crosses the prime meridian into the Eastern hemisphere.

The one-port shunt $R L C$ network in Fig. 10 exhibits negative differential-phase group delay (reflection response) when $R<Z_{o}$ [3].

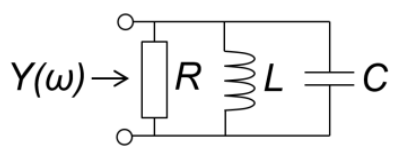

Fig. 10. Shunt $R L C$ network exhibiting negative differential-phase group delay (reflection response).

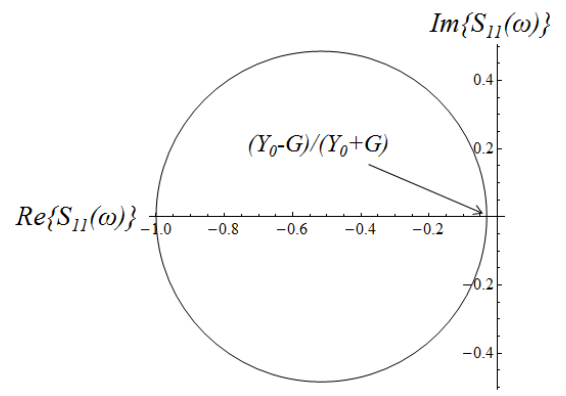

Fig. 11. Voltage-wave reflection coefficient $S_{11}(\omega)$ loop on a 2-D Smith chart for the one-port shunt $R L C$ network given in Fig. 10, having a zero S-parameter winding number when $R<Z_{o}$, exhibiting negative differentialphase group delay.

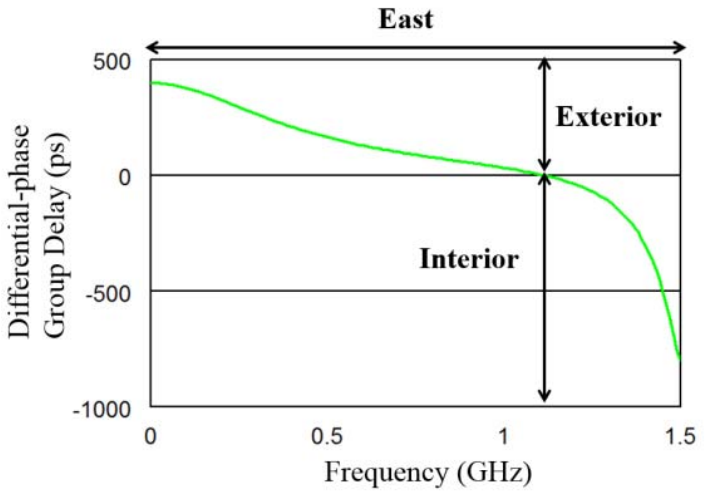

Fig. 12. Calculated differential-phase group delay (reflection response) for the one-port network given in Fig. 10.

From Fig. 10, the voltage-wave reflection coefficient is given as

$$
S_{11}(\omega)=\frac{Y_{0}-Y(\omega)}{Y_{0}+Y(\omega)}
$$

where $Y_{0}=1 / Z_{0}$ and $Y(\omega)$ is the driving point admittance of the network, given by

$$
Y(\omega)=G+j\left(\omega C-\frac{1}{\omega L}\right)
$$

where $G=1 / R$. It can be seen geometrically that (18) will map (19) into a circle onto the 2-D Smith chart, this time passing through $S_{11}\left(\omega_{o}\right)=\left(Y_{0}-G\right) /\left(Y_{0}+G\right)$ and $S_{11}(\omega)=-1$ at dc and infinite frequency. For example, with arbitrary values of $L=10 \mathrm{nH}, C=1 \mathrm{pF}$, and $R=47 \Omega$, with $Z_{0}=50 \Omega$, the calculated $S_{11}(\omega)$ and associated differential-phase group delay are shown in Figs. 11 and 12, respectively.

Fig. 13(a) shows a section of the calculated loop of $S_{11}(\omega)$ in the 2-D Smith chart, while Fig. 13(b) shows the corresponding differential-phase group delay with the 3-D Smith chart. 


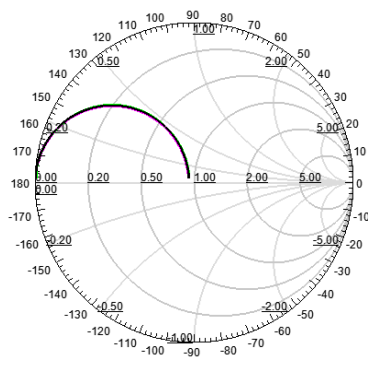

(a)

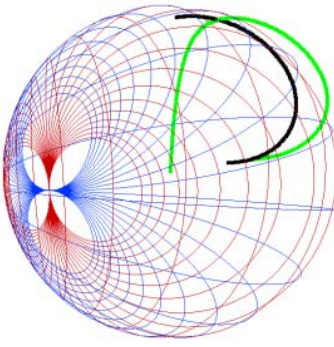

(b)
Fig. 13. Smith chart displays of calculated voltage-wave reflection coefficient (surface curve) for the network in Fig. 10. (a) 2-D chart with $S_{11}(\omega)$ corresponding to Fig. 11. (b) 3-D chart with $S_{\mathbf{1 1 3}}(\boldsymbol{\omega})$ and reflection response $\tau_{3 D}(\omega)$ curve (3-D space curve).

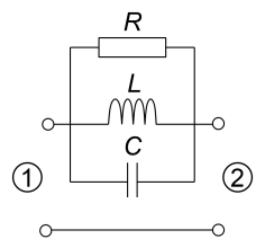

Fig. 14. Two-port series network with parallel $R L C$ tuned circuit exhibiting negative differential-phase group delay (transmission response).

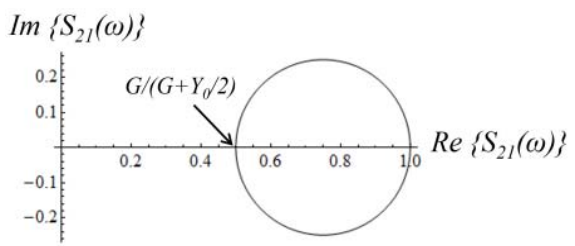

Fig. 15. Voltage-wave transmission coefficient $S_{21}(\omega)$ loop on a 2-D Smith chart for the two-port network shown in Fig. 14, having a zero S-parameter winding number, exhibiting negative differential-phase group delay.

As predicted by (7) and seen in Fig. 12, the $\tau_{3 D}(\omega)$ curve is interior from 1.12 to $1.5 \mathrm{GHz}$. Moreover, the movement with frequency of $\tau_{3 D}(\omega)$ starts above the Eastern hemisphere and tends toward the prime meridian; it never crosses, since the network is inductive in the displayed frequency range.

The two-port series network with parallel $R L C$ tuned circuit shown in Fig. 14 can also exhibit negative differential-phase group delay (transmission response) [3].

From Fig. 14, the voltage-wave transmission coefficient is given as

$$
S_{21}(\omega)=\frac{G+j\left(\omega C-\frac{1}{\omega L}\right)}{\left(G+Y_{0} / 2\right)+j\left(\omega C-\frac{1}{\omega L}\right)} .
$$

It can be seen that (20) maps $\omega$ into a circle onto the 2-D Smith chart; this time passing through $S_{21}\left(\omega_{o}\right)=G /\left(G+Y_{0} / 2\right)$ and $S_{21}(\omega)=1$ at dc and infinite frequency. For example, with arbitrary values of $L=3 \mathrm{nH}, C=5 \mathrm{pF}$, and $R=100 \Omega$, with $Z_{0}=50 \Omega$, the calculated $S_{21}(\omega)$ and associated differentialphase group delay are shown in Figs. 15 and 16, respectively.

Fig. 17(a) shows a section of the calculated loop of $S_{21}(\omega)$ in the 2-D Smith chart, while Fig. 17(b) shows the corresponding differential-phase group delay with the 3-D

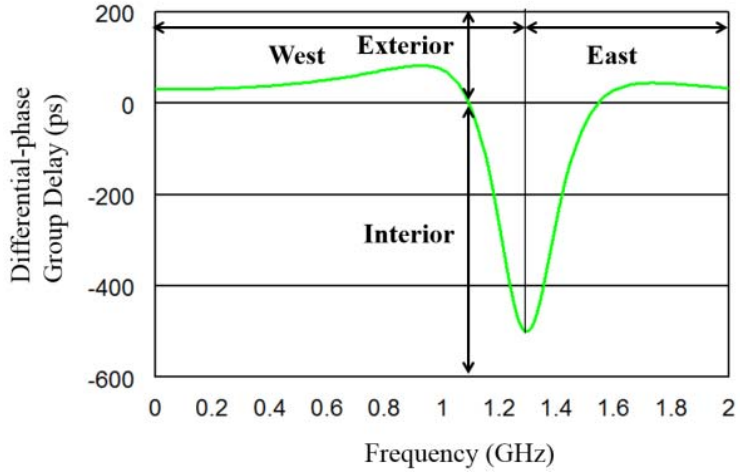

Fig. 16. Calculated differential-phase group delay (transmission response) for the parallel $R L C$ network given in Fig. 14.

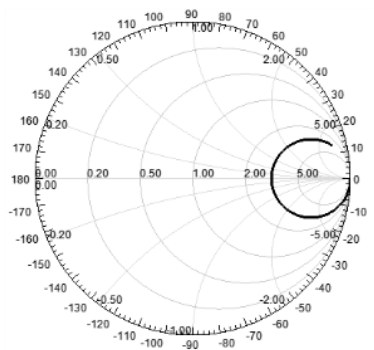

(a)

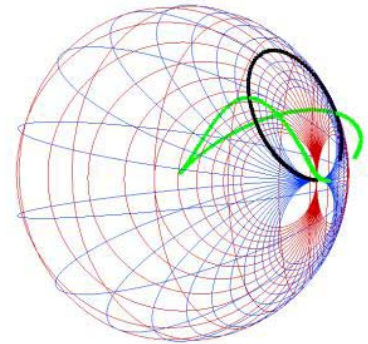

(b)
Fig. 17. Smith chart displays of calculated voltage-wave transmission coefficient (surface curve) for the network in Fig. 14. (a) 2-D chart with $S_{21}(\omega)$ corresponding to Fig. 15. (b) 3-D chart with $\boldsymbol{S}_{\mathbf{2 1 3}}(\boldsymbol{\omega})$ and transmission response $\tau_{3 D}(\omega)$ curve (3-D space curve).

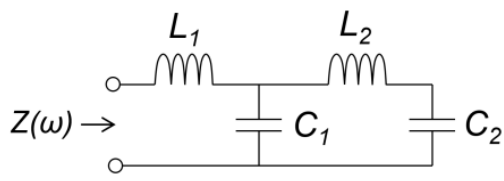

Fig. 18. Typical $L C$ ladder network exhibiting negative differential-phase group delay (reflection response) with positive S-parameter winding number for negative values of inductance and capacitance.

Smith chart. As predicted by (7) and seen in Fig. 16, the $\tau_{3 D}(\omega)$ curve is interior from 1.09 to $1.5 \mathrm{GHz}$. Moreover, below, at, and above at the undamped resonance frequency of $1.299 \mathrm{GHz}$, the network changes from being capacitive to resistive to inductive, respectively. As a result, the movement with frequency of $\tau_{3 D}(\omega)$ starts above the Western hemisphere crosses the prime meridian into the Eastern hemisphere.

\section{Positive S-Parameter Winding Number}

Let us now consider a one-port non-Foster network with negative valued inductances and capacitances [14], as shown in Fig. 18 (subscript $N F$ represents the non-Foster condition).

As recently shown [14], the driving point reactance/susceptance for a pure non-Foster network (made from ideal negative inductive and capacitive element values) is a strongly real function of negative type of real frequency. Thus, the circuit behaves like an ideal non-Foster network having negative reactance and susceptance frequency 


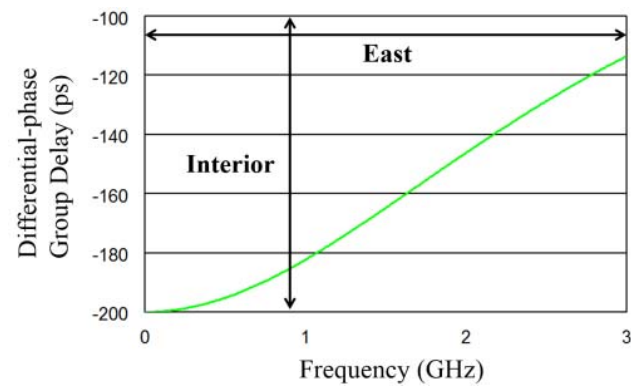

Fig. 19. Calculated differential-phase group delay (reflection response) for the $L C$ ladder network given in Fig. 18.

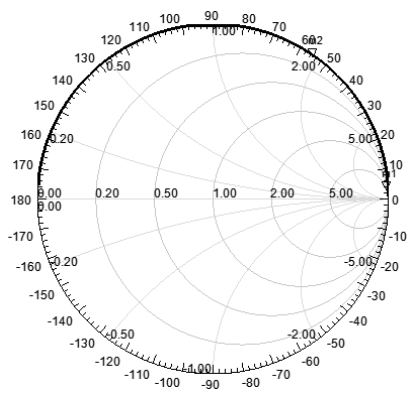

(a)

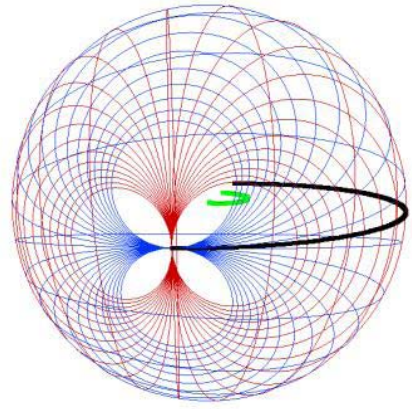

(b)
Fig. 20. Smith chart displays of calculated voltage-wave reflection coefficient (surface curve) for the network in Fig. 18. (a) 2-D chart with $S_{11}(\omega)$. (b) 3-D chart with $S_{113 D}(\omega)$ and reflection response $\tau_{3 D}(\omega)$ curve (3-D space curve).

gradients. In this case, $S_{11}(\omega)$ will rotate counterclockwise completely around the origin (having a positive S-parameter winding number) and exhibit a negative reflection response group delay. Using the following arbitrary values of $L_{1}=L_{2}=-1 \mathrm{nH}$ and $C_{1}=C_{2}=-1 \mathrm{pF}$, with $Z_{0}=50 \Omega$, the calculated differential-phase group delay is shown in Fig. 19.

Fig. 20(a) shows a section of the calculated loop of $S_{11}(\omega)$ in the 2-D Smith chart, while Fig. 20(b) shows the corresponding differential-phase group delay with the 3-D Smith chart.

\section{Filter EXAMPLES}

\section{A. Optimization of Reflection Response Group Delay}

In filter synthesis, one may use the Group Delay Method for determining the input coupling of the single input or output resonator, with all other resonators removed, based on voltagewave reflection coefficient analysis [24]. It is desirable to find the value of reflection response group delay at resonance, which is related to the external quality factor $Q_{e}$ [24]. As an example, consider the layout of the lossless combline crosscoupled bandpass filter shown in Fig. 21 [25].

The input coupling disk diameter and its position are optimized to peak the reflection response group delay with a $0 \mathrm{~dB}$ return loss (for a purely reactive resonator), at a target center frequency of $1.85 \mathrm{GHz}$ (corresponding to a target value set for $Q_{e}$ [24]). This is achieved through optimization, with the optimized target result shown in Fig. 22(a). With a Cartesian plot it is difficult to perform multiobjective goal

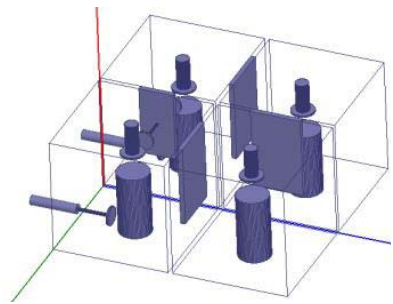

(a)

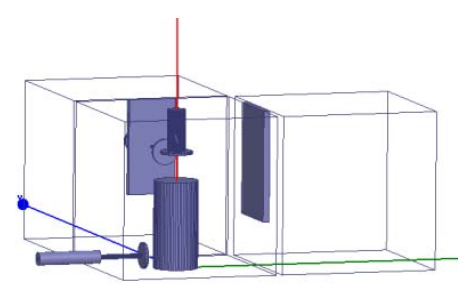

(b)
Fig. 21. Combline cross-coupled bandpass filter [25]. (a) Complete layout and (b) close-in view of one quadrant for calculating $Q_{e}$ by including the effects of the iris coupling and terminating reference impedance for the oneport network with all other resonators removed.

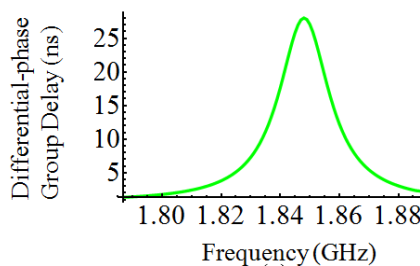

(a)

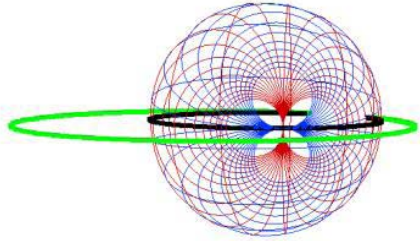

(b)
Fig. 22. Target synthesized reflection response group delay for the layout in Fig. 21(b). (a) Cartesian plot of target group delay $\tau_{T}(\omega)$. (b) 3-D Smith chart showing the target $S_{113 D T}(\omega)$ (surface curve) and $\tau_{3 D T}(\omega)$ (3-D space curve) responses.

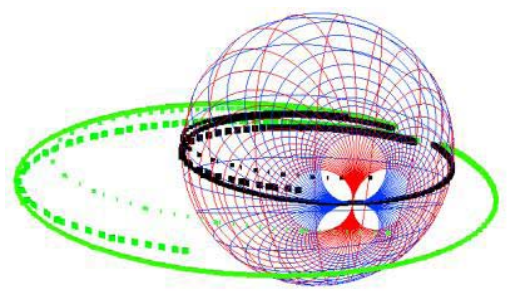

Fig. 23. Initial (dotted curves) and final (dashed curves) optimization results for both $S_{113 D}(\omega)$ and $\tau_{3 D}(\omega)$ to achieve the target (solid curves) for $S_{113 D T}(\omega)$ and $\tau_{3 D T}(\omega)$, shown in Fig. 22(b).

optimization (e.g., obtain a target group delay of $28.3 \mathrm{~ns}$ at a center frequency of $1.85 \mathrm{GHz}$ and the voltage-wave reflection coefficient on the unity radius contour of the standard 2-D Smith chart or equator of the 3-D Smith chart). However, on the 3-D Smith chart, the target values of S-parameters and associated differential-phase group delay can be combined onto one visualization platform. In this way, it is possible to optimize not only the disk diameter and its position but also the input coaxial cable dimensions, detect the presence of any additional losses, and view distances from multiple goals over the desired frequency range, at each optimization step.

Using the 3-D Smith chart, the associated differential-phase group delay can now be simultaneously displayed with the S-parameters. For example, Fig. 23 displays the initial (dotted curves) and final (dashed curves) optimization results for both $S_{113 D}(\omega)$ and $\tau_{3 D}(\omega)$ to achieve the target (solid curves) for $S_{113 D T}(\omega)$ and $\tau_{3 D T}(\omega)$, shown in Fig. 22(b). Both dotted curves are distant from their respective target solid curves, whereas the dashed curves are close. It should be noted that both qualitative and quantitative assessments are much easier 


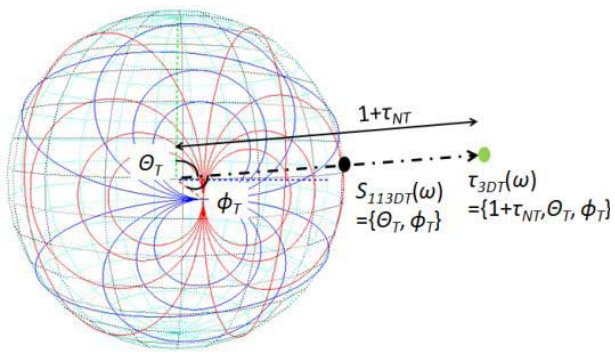

Fig. 24. $\tau_{3 D T}(\omega)$ represented in a spherical coordinate system. At each unique value of $\omega$, the corresponding value of $S_{113 D T}(\omega)$ can be read-off from the surface of the 3-D Smith chart.

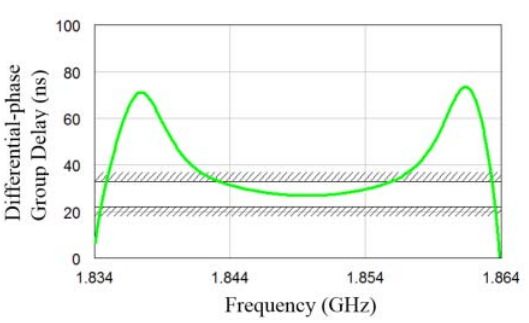

(a)

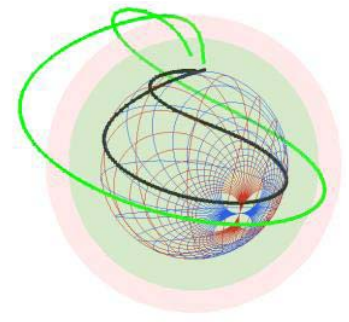

(b)
Fig. 25. Setting target masks for the differential-phase group delay in an advanced optimization step of a filter design [26]. (a) Cartesian plot. (b) 3-D Smith chart $S_{213 D}(\omega)$ (surface curve) and $\tau_{3 D}(\omega)$ (3-D space curve). The 3-D target masks are represented by two transparent concentric spheres.

with user-interactive viewing of the 3-D Smith chart, rather than being seen in the static 2-D images shown here.

Unlike with a $2-D$ curve, $\tau_{3 D}(\omega)$ is a space curve defined by the homothety in (7). With reference to Fig. 24, $\tau_{3 D T}(\omega)$ can be expressed with spherical coordinates, relative to the origin of the Riemann sphere, with $\omega$ being the frequency of interest.

The reflection response group delay $\boldsymbol{\tau}_{3 D T}(\omega)$ is mathematically represented by (21), with (22) corresponding to the associated S-parameter $S_{113 D T}(\omega)$ in the spherical coordinate system

$$
\boldsymbol{\tau}_{3 D T}(\omega)=\left\{\left(1+\tau_{N T}\right), \Theta_{T}, \phi_{T}\right\}
$$

and

$$
S_{113 D T}(\omega)=\left\{\Theta_{T}, \phi_{T}\right\}
$$

where $\left(1+\tau_{\mathrm{NT}}\right)$ is the distance from the center of the sphere, $\Theta_{T}$ is the target colatitude, and $\phi_{T}$ is the target longitude. By fitting $\tau_{3 D}(\omega)$, given as

$$
\boldsymbol{\tau}_{3 D}(\omega)=\left\{\left(1+\tau_{N}\right), \Theta, \phi\right\}
$$

with (21), it can be seen that $\left(1+\tau_{N}\right)=\left(1+\tau_{N T}\right), \Theta=\Theta_{T}$, $\phi=\phi_{T}$ and thus

$$
S_{113 D}(\omega)=\{\Theta, \phi\} \equiv S_{113 D T}(\omega) .
$$

This means that, with the Group Delay Method, once the reflection response group delay $\tau_{3 D}(\omega)$ is made to fit $\tau_{3 D T}(\omega)$ at each unique frequency point then the associated S-parameter $S_{113 D}(\omega)$ will automatically fit $S_{113 D T}(\omega)$ and, therefore, the complete optimization process can be achieved by a single

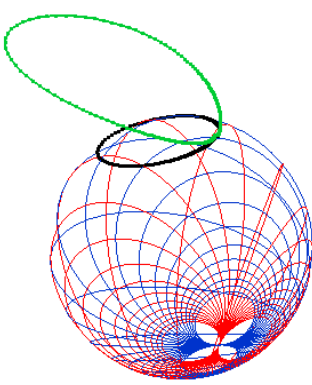

(a)

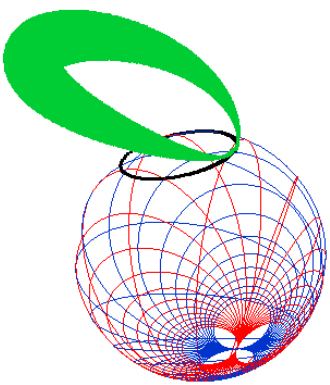

(b)
Fig. 26. 3-D Smith chart displays of calculated $S_{\mathbf{2 1 3}}(\boldsymbol{\omega})$ (surface curve) for an arbitrary lossy two-port resonator with (a) $\tau_{3 D}(\omega)$ (3-D space curve) and (b) resonator loaded quality factor $Q_{3 D}(\omega, \theta)$ (3-D space generalized cylinder).

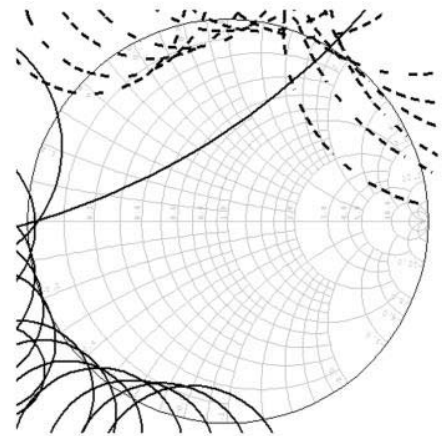

(a)

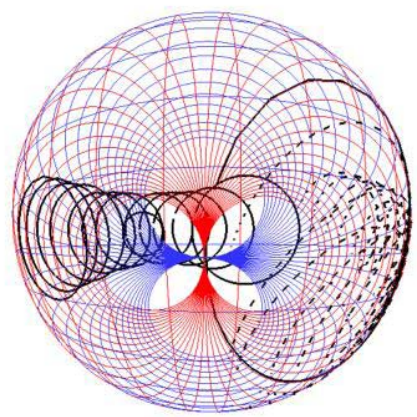

(b)
Fig. 27. Smith chart displays of source (solid) and load (dashed) stability circles for a Motorola 2N6679A bipolar transistor at different frequencies (0.1 and 0.5 to $6.5 \mathrm{GHz}$, in $0.5 \mathrm{GHz}$ steps) [30]. (a) 2-D chart. (b) 3-D chart.

3-D parametrical curve fitting, effectively disregarding the associated S-parameter curve in this optimization process.

\section{B. Equalization of Transmission Response Group Delay}

The flatness of group delay across the operating bandwidth of a filter [26], [27] is an important design requirement for many applications. On a conventional Cartesian plot of $\tau(\omega)$, target masks are normally introduced to indicate its minimum and maximum allowable values, as seen in Fig. 25(a). With a 3-D Smith chart, two transparent concentric spheres can be introduced, each representing the minimum and maximum allowable values, as shown in Fig. 25(b). For example, in Fig. 25(b), $\tau_{3 D}(\omega)$ lying within the green and orange spheres, in proximity to the undamped resonance frequency, obeys the target mask constraints given in Fig. 25(a), while it is still possible to visualize $S_{\mathbf{2 1 3}}(\boldsymbol{\omega})$ on the surface of the 3-D Smith chart.

\section{Loaded Resonator Quality Factor}

An important target in the design of filters is the quality factor of its resonators, which is limited by available technological constraints. The 3-D Smith chart allows for the simultaneous display of differential-phase group delay and associated loaded resonator quality factor and S-parameters. 
As an arbitrary example, a two-port lossy resonator was synthesized using electromagnetic field simulation software. With a reference impedance $Z_{0}=50 \Omega$, the transmission response group delay is $14.8 \mathrm{~ns}$. Using (13), the equivalent series $R L C$ resonator's loaded Q-factor is 200 at its undamped resonance frequency of $4.3 \mathrm{GHz}$. The results are displayed in Fig. 26, from 4.0 to $4.6 \mathrm{GHz}$. As with all the results displayed with the 3-D Smith chart, in practice, markers display the quantitative numerical values associated with all the curves.

\section{CONCLUSION}

This paper presents a new way of simultaneously displaying various parameters with a 3-D Smith chart. Here, mathematical concepts, inspired from elementary differential geometry and topology, are used to implement 3-D projections. The mathematical relationship between S-parameter winding number and differential-phase group delay is defined, and a condition for a circuit to exhibit both positive and negative differential-phase group delay is given. The associated loaded resonator quality factor is also displayed for the first time with the 3-D Smith chart.

A variety of different types of networks (NGD, nonFoster and filter) were arbitrarily chosen as exemplars. With reflection response group delay optimization, it was shown that 3-D curve fitting of the differential-phase group delay automatically satisfies the associated S-parameter requirement (modulus and phase). Here, two target responses are optimized by a single 3-D curve fit. Finally, the convenience of being able to simultaneously display a wider range of parameters on one visualization platform, with the 3-D Smith chart, may help to speed-up the design and analysis of microwave circuits by the user.

\section{APPENDIX \\ 3-D SMith ChaRt Display Enhancements}

Negative resistance represents a challenge for the 2-D Smith chart [28] (due to scaling problems with large negative resistance values); while geometrical shape distortions map onto the new hyperbolic Smith chart [29] (circles on the 2-D Smith chart may lose their shape on the hyperbolic version of the Smith chart). These limitations are avoided with a 3-D Smith chart, since infinity converges to a convenience point on the Riemann sphere (South Pole), while no shape distortions occur (circles on a 2-D Smith chart are always circles on a 3-D Smith chart).

In order to make the 3-D Smith chart a practical tool, several display enhancements have been developed to help in the design and analysis of microwave circuits [12]. For example, input and output stability circles have been recently introduced. To avoid oscillation, the stability analysis of an amplifier must be performed over a wide frequency range $[10,30]$. The current 2016 version of the 3-D Smith chart [12] can now simultaneously plot stability circles, as shown in Fig. 27, using measured S-parameters (at 0.1 and from 0.5 to $6.5 \mathrm{GHz}$, in $0.5 \mathrm{GHz}$ steps) for the Motorola 2N6679A bipolar transistor [30]. In order to achieve unconditional stability, the amplifier must be designed so that all the stability circles lie within the Southern hemisphere of the 3-D Smith chart. Indeed, as seen in Fig. 27(b), all the circles can be viewed in their entirety [(without the scaling issues required with Fig. 27(a)] and having no shape distortion.

\section{ACKNOWLEDGMENT}

The authors would like to thank Prof. D. Dascalu, University Politehnica of Bucharest, Romania, for the support given during the early development of this paper.

\section{REFERENCES}

[1] S. Lucyszyn, I. D. Robertson, and A. H. Aghvami, "Negative group delay synthesizer," Electron. Lett., vol. 29, no. 9, pp. 798-800, Apr. 1993.

[2] S. Lucyszyn and I. D. Robertson, "Analog reflection topology building blocks for adaptive microwave signal processing applications," IEEE Trans. Microw. Theory Techn., vol. 43, no. 3, pp. 601-611, Mar. 1995.

[3] H. Mirzaei and G. V. Eleftheriades, "Realizing non-foster reactive elements using negative-group-delay networks," IEEE Trans. Microw. Theory Techn., vol. 61, no. 12, pp. 4322-4332, Dec. 2013.

[4] H. Mirzaei, "Negative-group-delay and non-Foster electromagnetic structures," Ph.D. dissertation, Dept. Elect. Comput. Eng., Toronto Univ., Toronto, ON, Canada, 2015.

[5] B. Ravelo, "First-order low-pass negative group delay passive topology," IET Electron. Lett., vol. 52, no. 2, pp. 124-126, Jan. 2016.

[6] G. Chaudhary, Y. Jeong, and J. Im, "A design of reconfigurable negative group delay circuit without external resonators," IEEE Antennas Wireless Propag. Lett., vol. 14, pp. 883-886, 2015.

[7] C.-T. M. Wu and T. Itoh, "Maximally flat negative group-delay circuit: A microwave transversal filter approach," IEEE Trans. Microw. Theory Techn., vol. 62, no. 6, pp. 1330-1342, Jun. 2014.

[8] W. J. Otter, S. M. Hanham, N. Klein, and S. Lucyszyn, "Millimeterwave negative group delay network," in Proc. Union Radio-Sci. Int. Asia-Pacific Radio Sci. Conf. (URSI AP-RASC), Seoul, South Korea, Aug. 2016, pp. 1205-1207.

[9] A. A. Muller, P. Soto, D. Dascalu, D. Neculoiu, and V. E. Boria, "A 3-D Smith chart based on the Riemann sphere for active and passive microwave circuits," IEEE Microw. Compon. Lett., vol. 21, no. 6, pp. 286-288, Jun. 2011.

[10] A. A. Müller, P. Soto, D. C. Dascalu, and V. E. Boria, "The 3D Smith chart and its practical applications," Microw. J., vol. 5, no. 7, pp. 64-74, Jul. 2012.

[11] A. A. Muller, P. Soto, A. Moldoveanu, V. Asavei, and V. E. Boria, "A visual comparison between voltage and power wave reflection coefficients of microwave circuits," in Proc. Asia-Pacific Microw. Conf. Kaohsiung, Taiwan, Dec. 2012, pp. 1259-1261.

[12] A. A. Muller, "3D Smith Chart," Aug. 2016. [Online]. Available: http://www.3dsmithchart.com

[13] A. A. Muller et al., "Apollonius unilateral transducer constant power gain circles on 3D Smith charts," IET Electron. Lett., vol. 50, no. 21, pp. 1531-1533, Oct. 2014.

[14] A. A. Muller and S. Lucyszyn, "Properties of purely reactive Foster and non-Foster passive networks," IET Electron. Lett., vol. 51, no. 23 , pp. 1882-1884, Nov. 2015.

[15] A. N. Pressley, Elementary Differential Geometry. New York, NY, USA: Springer, 2012

[16] P. D. McSwiggen, "Calculus course, lab 9-Space curves," Dept. Math. Sci., Univ. Cincinnati, Cincinnati, OH, USA, Tech. Rep., 2002. [Online]. Available: https://math.uc.edu/ mcswgn/Calc3Lab/lab9.pdf

[17] I. Neulander, "Rendering generalized cylinders using the A-buffer," M.S. thesis, Dept. Comput. Sci., Univ. Toronto, Toronto, ON, Canada, 1997.

[18] I. Neulander and M. Van de Panne, "Rendering generalized cylinders with paintstrokes," in Proc. Graph. Interface, 1998, pp. 233-242.

[19] B. E. Meserve, Fundamental Concepts of Geometry. Reading, MA, USA: Addison-Wesley, 1955, pp. 163-169.

[20] G. B. Thomas, M. D. Weir, and J. R. Haas, "Vectors and the geometry of space," in Thomas' Calculus, 12th ed. Boston, MA, USA: Pearson, 2001, pp. 660-706. 
[21] W. J. Otter, S. M. Hanham, N. M. Ridler, G. Marino, N. Klein, and S. Lucyszyn, "100 GHz ultra-high $Q$-factor photonic crystal resonators," Sens. Actuators A, Phys., vol. 217, pp. 151-159, Sep. 2014.

[22] R. Rostamian, "The differential geometry of tube plots and computer graphics," Dept. Math. Statist., Univ. Maryland, Baltimore MD, USA, Tech. Rep., 2000. [Online]. Available: http://userpages.umbc.edu/ rostamia/pubs/tubeplot.pdf

[23] T. Sheil-Small, "The degree principle and the fundamental theorem of algebra," in Complex Polynomials, 1st ed. Cambridge, U.K.: Cambridge Univ. Press, 2002, pp. 26-40.

[24] R. J. Cameron, C. M. Kudsia, and R. R. Mansour, "Design and physical realization of coupled resonator filters," in Microwave Filters for Communication Systems, Fundamentals, Design and Applications, 1st ed. Hoboken, NJ, USA: Wiley, 2007, pp. 515-520.

[25] A. A. Muller, J.-F. Favennec, and E. Sanabria-Codesal, "Coupling matrix filter synthesis based on reflection matrices," in Proc. Asia-Pacific Microw. Conf., Nanjing, China, Dec. 2015, pp. 1-3.

[26] A. A. Muller, A. Moldoveanu, V. Asavei, E. Sanabria-Codesal, and J. F. Favennec, "Lossy coupling matrix filter synthesis based on hyperbolic reflections," in IEEE MTT-S Int. Microw. Symp. Dig., San Francisco, CA, USA, May 2016, pp. 1-4.

[27] $\mathrm{H}$. Hu and K.-L. Wu, "A generalized coupling matrix extraction technique for bandpass filters with uneven-Qs," IEEE Trans. Microw. Theory Techn., vol. 62, no. 2, pp. 244-251, Feb. 2014.

[28] P. H. Smith, "Transmission-line calculator," Electronics, vol. 12, pp. 29-31, Jan. 1939

[29] A. A. Muller and E. Sanabria-Codesal, "A hyperbolic compact generalized Smith chart," Microw. J., vol. 59, no. 8, pp. 90-94, Aug. 2016.

[30] J. F. White, High Frequency Techniques: An Introduction to RF and Microwave. Hoboken, NJ, USA: Wiley, 2004.

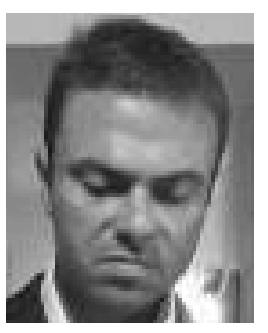

Andrei A. Muller (GS'06-M'07) received the Ph.D. degree in telecommunications engineering from the University Politehnica of Bucharest, Bucharest, Romania, in 2011.

During his Ph.D. degree, he was involved in several collaborative projects in network design (Germany), antenna design (South Africa), and pure mathematics (Spain). In 2012, he held a post-doctoral position with Labsticc-CNRS, Brest, France.

Dr. Muller was a recipient of the Gheorghe Cartianu Award of the Romanian Academy for the 2011 IEEE MICROwAVE AND Wireless COMPONENTS LETTERS, "A 3-D Smith Chart Based on the Riemann Sphere for Active and Passive Microwave Circuits" in 2013. He received a four-year Marie Curie Integration Grant Fellowship from the European Union while with the Microwave Applications Group, Polytechnic University of Valencia, Valencia, Spain, in 2013. Since 2016, he has been an Associate Editor of IEEE ACCESS.

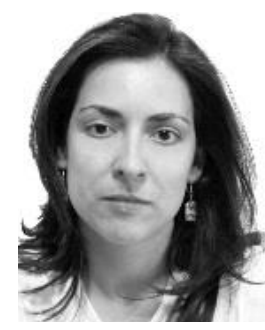

Esther Sanabria-Codesal received the Ph.D. degree in geometry and topology from the University of Valencia, Valencia, Spain, in 2002.

She is an Assistant Professor with the Department of Applied Mathematics, Polytechnic University of Valencia, Valencia. Her current research interests include theory of singularities applied to geometry and mathematical modeling based on graphs.

Dr. Sanabria-Codesal has participated in numerous research projects and conferences.

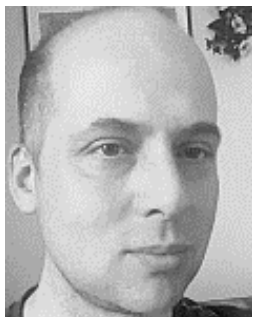

Alin Moldoveanu is currently a Full Professor and a Faculty Member of automatic control and computers with the Computer Science and Engineering Department, University Politehnica of Bucharest, Bucharest, Romania, where he teaches software engineering and virtual reality. $\mathrm{He}$ is the Director and is responsible for several national and European research projects in the areas of virtual and augmented reality, eHealth, and eLearning. His current research interests include virtual and augmented reality (exploring and applying immersion, sensory substitution, and distorted reality), eHealth (assistive and rehabilitative solutions, prevention of hospital acquired infections), and eLearning (3-D MMO mixed-reality campuses).

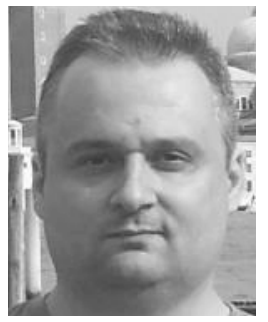

Victor Asavei received the Ph.D. degree in computer science and information technology from the University Politehnica of Bucharest, Bucharest, Romania, in 2011.

$\mathrm{He}$ is currently an Assistant Professor (Lecturer) and a faculty member of automatic control and computers with the Computer Science and Engineering Department, University Politehnica of Bucharest. He has co-authored approximately 60 papers and 4 books in the fields of computer graphics, distributed computing, software engineering, and medical ITs and has participated in numerous national and international research projects. His current research interests include real-time computer graphics and general-purpose computing on graphics processing units.

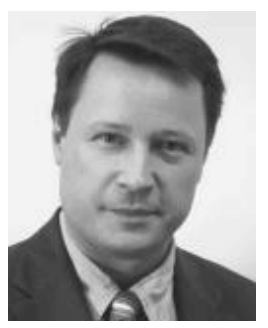

engineering.

Prof. Lucyszyn has delivered many invited presentations at international conferences. He is a Fellow of the Institution of Electrical Engineers (U.K.), the Institute of Physics (U.K.), and the Electromagnetics Academy (USA). He was an Associate Editor of the IEEE/ASME JOURNAL OF MicRoeleCtromeCHANICAL SySTEMS from 2005 to 2009. He was the Chairman of the 41st European Microwave Conference, Manchester, U.K., in 2011. In 2009, he was appointed as an IEEE Distinguished Microwave Lecturer (DML) from 2010 to 2012 and an Emeritus DML in 2013. He is currently a European Microwave Lecturer for the European Microwave Association 